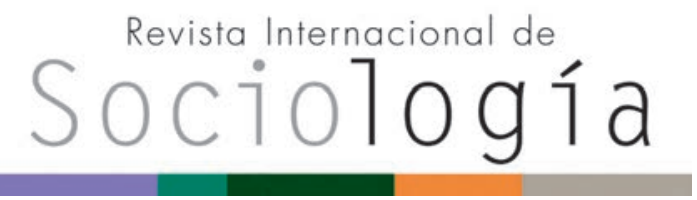

Revista Internacional de Sociología RIS

vol. 76 (2), e097, abril-junio, 2018, ISSN-L:0034-9712

https://doi.org/10.3989/ris.2018.76.2.16.01220

\section{DIFERENCIAS Y SIMILITUDES EN LAS DECISIONES LABORALES $Y$ FAMILIARES DE LAS MUJERES EN ESPAÑA Y PORTUGAL}

\author{
NuRIA LEgAZPE MoRALEJA \\ Universidad de Castilla-La Mancha, España \\ Nuria.Legazpe@uclm.es \\ ORCID iD: https://orcid.org/0000-0002-2161-3768
}

Cómo citar este artículo / Citation: Legazpe Moraleja, N. 2018. "Diferencias y similitudes en las decisiones laborales y familiares de las mujeres en España y Portugal". Revista Internacional de Sociología 76(2):e097. https:// doi.org/10.3989/ris.2018.76.2.16.01220

\section{RESUMEN}

El objetivo del presente trabajo es analizar qué factores influyen en las decisiones laborales, de convivencia en pareja y de no ser madres de las mujeres trabajadoras en España y Portugal. Para alcanzar este objetivo se ha explotado la Encuesta de la Unión Europea sobre Condiciones de Vida. La estrategia empírica ha consistido en un modelo probit trivariante, lo que permite estimar las tres variables dependientes de manera simultánea. Los resultados muestran que la edad, el país de nacimiento, el estado de salud y el año de la entrevista tienen un impacto distinto en las mujeres españolas respecto de las mujeres portuguesas.

\section{Palabras Clave}

Convivencia; No maternidad; Participación laboral; Probit trivariante.

\section{DIFFERENCES AND SIMILARITIES IN WOMEN'S DECISIONS ON LABOR AND FAMILY IN SPAIN AND PORTUGAL}

Copyright: () 2018 CSIC. Este es un artículo de acceso abierto distribuido bajo los términos de la licencia de uso y distribución Creative Commons Reconocimiento 4.0 Internacional (CC BY 4.0).

Recibido: 03/08/2016. Aceptado: 15/11/2017

\section{Abstract}

The aim of this paper is to analyse the changes along the period 2005-2013 in the determinants of the decision to participate in the labour market, union formation decisions and non-maternity decisions of working women in Spain and Portugal. To that aim, we use the European Union Statistics on Income and Living Conditions Survey. The econometric technique is a trivariate probit model, which allows estimating the three dependent variables simultaneously. The results show, among other things, that age, country of birth, health status and year of the interview have a different impact on Spanish women compared to Portuguese women.

\section{KEYWORDS}

Labour participation; Non-maternity; Probit trivariate; Union formation. 


\section{INTRODUCCIÓN}

El proceso de modernización social, económica y cultural de las sociedades industriales se ha visto acompañado por un mayor acceso de las mujeres a todos los niveles educativos y a la formación. Estos cambios en la educación han jugado un papel preponderante en la evolución de las mujeres en las sociedades occidentales, ya que ha influido de manera directa en sus decisiones laborales y familiares $y$, más concretamente, en el momento en el que deciden iniciar una convivencia en pareja, tener hijos, y el número de hijos que deciden tener. El aumento del nivel educativo de las mujeres europeas en las últimas décadas les permite obtener mayores rentas laborales, resultado de su mayor productividad en el sistema productivo (Schultz 1960; Becker 1964). De hecho, las tasas de ocupación femenina han experimentado aumentos en toda Europa, incluidos los países del sur de Europa, aunque dicho incremento ha sido más moderado. A día de hoy, los países del sur de Europa (España, Italia y Grecia) siguen presentando las tasas de ocupación femenina más reducidas de la UE-28. Portugal es la excepción al modelo mediterráneo, presentando tasas de ocupación femenina por encima de la media de la UE-15 (Figura 1), debido entre otras razones, a las dificultades que tienen las familias para llegar a fin de mes y que animan a las mujeres portuguesas a incorporarse al mercado laboral para contribuir a la economía familiar. La tasa de empleo femenino en Portugal es más similar a la de otros países nórdicos donde se han aplicado generosas y flexibles políticas familiares orientadas tanto a los hombres como a las mujeres con responsabilidades familiares con el objetivo de facilitar la conciliación de la vida familiar y laboral.

En la mayoría de los países occidentales, la mayor participación laboral femenina se ha visto acompañada por una reducción en el número de hijos debido al retraso de la primera maternidad. La fecundidad por debajo del nivel de reemplazo será probablemente una norma global en las próximas décadas; sin embargo, ya a principios de la década de los 90, países como España e Italia experimentaron mínimos históricos (Esping-Andersen 2013). Esto se relaciona con los diversos factores que impiden la conciliación de la vida familiar y laboral en los países del arco mediterráneo; factores como las peculiaridades del mercado laboral, con elevadas tasas de desempleo y acusada precariedad, la situación del mercado inmobiliario, y la falta de políticas públicas tendentes a atenuar estos efectos desfavorables, han conducido a que las tasas de fecundidad continúen en declive (Delgado et al. 2006). Si bien durante la fase expansiva de la economía se observa una recuperación de las tasas de fecundidad, en parte por el impacto de la llegada de inmigrantes que presentaban una estructura de edad más joven y una tasa de fecundidad más elevada, desde el inicio de la crisis económica, especialmente en España y Portugal, el número medio de hijos ha disminuido entre las mujeres de todas las edades, lo que significa una disminución global de la fecundidad (Figura 2).

El principal objetivo del presente trabajo es analizar los factores que influyen en las decisiones laborales, de convivencia en pareja y de no ser madres de las mujeres, realizando una comparativa entre España y Portugal, para vislumbrar si existen diferencias en dichas decisiones entre estos dos países pertenecientes al arco mediterráneo y que comparten analogías respecto a su historia, sistemas de valores y peculiaridades institucionales pero que, pese a su cercanía en muchos aspectos, presentan importantes diferencias en lo que respecta a las tasas de ocupación femenina y al porcentaje de hogares sin hijos. La evidencia empírica apunta a que las decisiones laborales y familiares de las mujeres están estrechamente relacionadas y a que hay determinantes comunes, como el nivel educativo o la edad, que hacen que las tres decisiones no sean

Figura 1.

Tasas de empleo femenino en España, Portugal y UE-15.

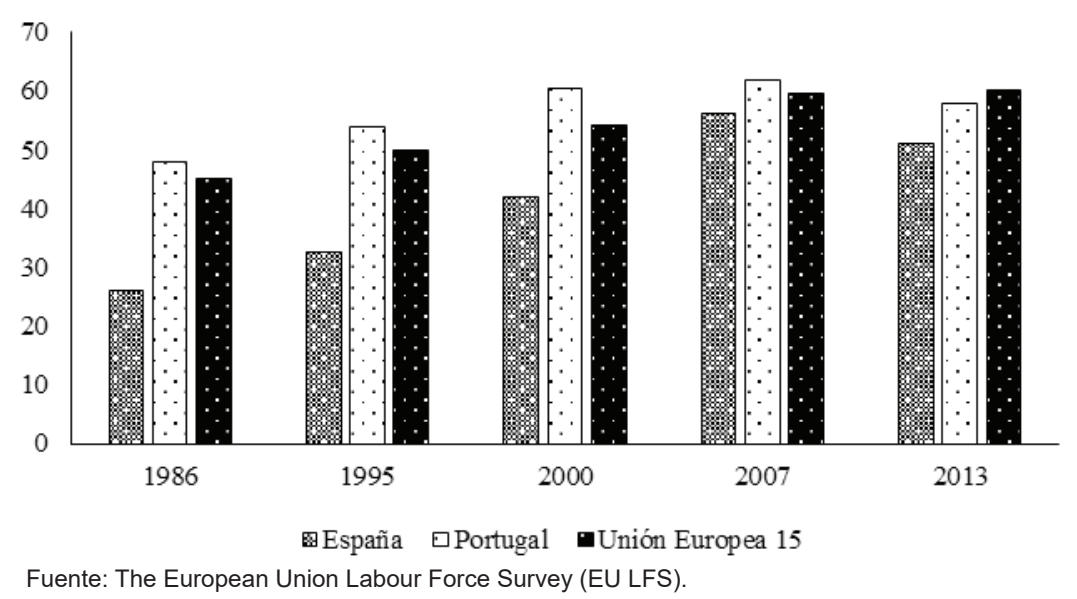

RIS [online] 2018, 76 (2), e097. REVISTA INTERNACIONAL DE SOCIOLOGÍA. ISSN-L: 0034-9712 
Figura 2.

Tasas de fecundidad en los países del sur de Europa (1986-2013).

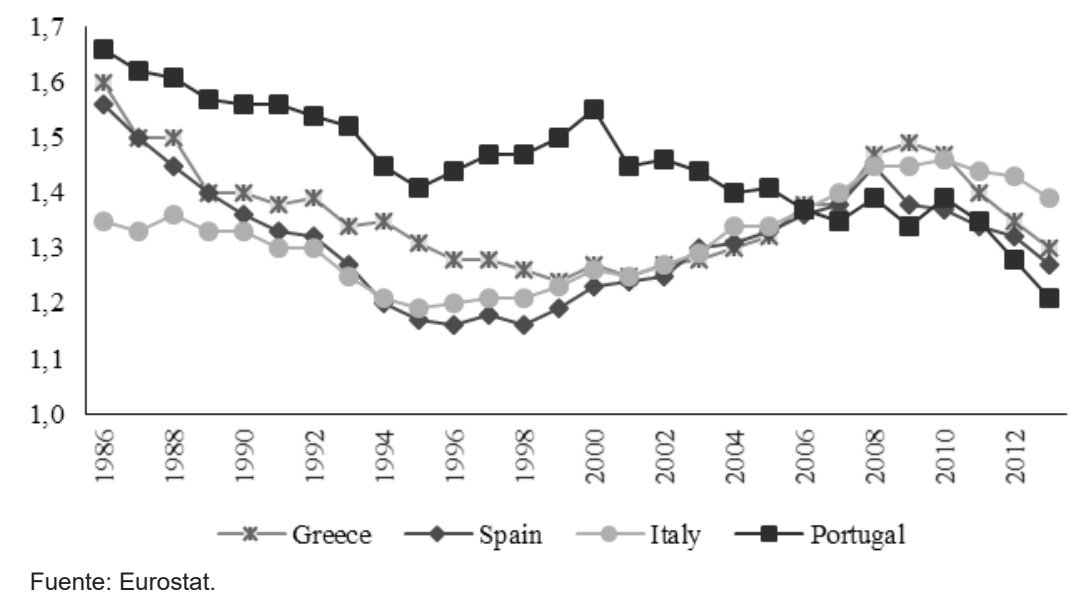

independientes. Por este motivo, la estrategia analítica se basará en la estimación de un modelo probit trivariante, lo cual permite incorporar determinantes comunes que afectan al mismo tiempo a las tres decisiones. Para llevar a cabo el análisis se utilizará la Encuesta de la Unión Europea sobre Condiciones de Vida (EU-SILC) realizada por Eurostat (2005-2013).

La estructura del trabajo es la siguiente. Tras esta introducción, en la sección 2 se realiza una descripción de las transformaciones habidas en las decisiones laborales y familiares de las mujeres en España y Portugal. En la sección 3 se recogen diversos enfoques teóricos. En la siguiente sección se presenta la base de datos y la metodología. La sección 5 se dedica a la presentación de los principales resultados obtenidos en la estimación del modelo probit trivariante. Por último, se presentan las conclusiones.

\section{TRANSFORMACIONES EN LAS DECISIONES LABORALES Y FAMILIARES}

Uno de los rasgos más característicos de los mercados de trabajo de los países desarrollados a partir de la segunda mitad del siglo XX ha sido el importante aumento de la presencia de la mujer en el empleo remunerado. Aunque las mujeres españolas, al igual que las de otros países del arco mediterráneo, han presentado sistemáticamente tasas de empleo muy por debajo de las de la media de la UE-15 (Tabla I), a partir de la segunda mitad de los años 80 se produce un cambio de tendencia. Así, la tasa de empleo femenina en España que era de un 31,7\% en 1995 aumentaba en casi veinte puntos porcentuales en dos décadas, alcanzando el 51,2\% en 2014. En Garrido (1993) se advierte que las mujeres nacidas a partir de la década de los 50 presentan patrones de empleo diferentes a sus antecesoras, dado que estas mujeres muestran un proceso de expansión educativa que supone un retraso en la incorporación al mercado de trabajo y a la maternidad y una menor tendencia a interrumpir sus carreras laborales para dedicarse en exclusiva al cuidado de la familia. Se trataba mayoritariamente de mujeres con nivel educativo superior que se empleaban en el sector sanitario, educativo y en la administración pública. A partir de la década de los 90, y en plena expansión del mercado de trabajo español, destaca la participación de las mujeres en el sector inmobiliario, la venta al por menor, la salud y el trabajo social y la hostelería y la restauración (Gónzales y Segales 2013). Se trata de empleos con una alta tasa de temporalidad y bajos salarios ${ }^{1}$ que son principalmente ocupados por mujeres con baja cualificación. Por tanto, en el caso de las mujeres españolas, se pone de manifiesto que aunque la mayor participación laboral se produce entre las mujeres con mayor nivel educativo, parece que, esta tendencia, en los últimos años, se ha extendido al resto de mujeres, con menor nivel educativo.

Tabla I.

Tasas de empleo femenino.

\begin{tabular}{|l|c|c|c|c|}
\hline & $\mathbf{1 9 9 5}$ & $\mathbf{2 0 0 0}$ & $\mathbf{2 0 0 7}$ & $\mathbf{2 0 1 4}$ \\
\hline España & $\mathbf{3 1 , 7}$ & $\mathbf{4 1 , 2}$ & $\mathbf{5 5 , 3}$ & $\mathbf{5 1 , 2}$ \\
\hline Grecia & 38,0 & 41,8 & 47,7 & 41,1 \\
\hline Italia & 35,5 & 39,3 & 46,6 & 46,8 \\
\hline Portugal & $\mathbf{5 4 , 3}$ & $\mathbf{6 0 , 5}$ & $\mathbf{6 1 , 8}$ & $\mathbf{5 9 , 6}$ \\
\hline Unión Europea 28 & - & - & $\mathbf{5 8 , 2}$ & $\mathbf{5 9 , 6}$ \\
\hline
\end{tabular}

Fuente: The European Union Labour Force Survey (EU LFS).

En cuanto a la participación laboral de las mujeres portuguesas, éstas han registrado una tasa de empleo por encima del $50 \%$, superando la media de la Unión Europea de los 28 (UE-128). Portugal presenta una estructura del mercado laboral peculiar, con una estricta regulación laboral, al igual que sucede en otros países sureños de Europa. Esta circunstancia ha tendido a sostener la seguridad en el empleo a tiempo completo de los participantes del mercado de trabajo a costa de menores probabilidades de empleo 
para los nuevos trabajadores y para las personas que buscan empleo a tiempo parcial (Borra 2010). En el mismo sentido, Ruivo et al. (1998) señalan que en Portugal y España el trabajo a tiempo parcial es, principalmente, involuntario, mientras que en otros países europeos se puede considerar una elección de la mujer. En países como España y Portugal la escasez de empleo a tiempo parcial en condiciones aceptables o favorables hace que sea difícil para las mujeres tener hijos y participar en el mercado laboral sin el apoyo de otros familiares (Legazpe 2016). En este contexto, ¿qué explica la mayor participación laboral de las mujeres portuguesas? El menor desarrollo del país, que se refleja en una reducida renta familiar, y los bajos salarios que reciben las familias portuguesas obligan inexorablemente a las mujeres a incorporarse al mercado laboral para contribuir con un segundo sueldo a la economía familiar. A esto hay que añadir que, durante años, las guerras coloniales mantuvieron a un gran número de militares alejados del país, así como el proceso migratorio a centro Europa, son los factores más determinantes en la explicación de las mayores tasas de participación laboral (Guerreiro et al. 2007).

El aumento de la participación femenina en el mercado de trabajo en las últimas décadas ha tenido un importante impacto en sus decisiones familiares. El patrón mediterráneo de formación familiar de finales del siglo XX se caracterizaba por una tardía emancipación residencial de los jóvenes, un retraso en la formación de la pareja, una difusión todavía limitada de la cohabitación y un alto grado de sincronización entre el abandono del hogar paterno, la formación de la primera unión y la primera maternidad (Baizán et al. 2003). En la tabla II se refleja un descenso de tasa bruta de nupcialidad²; en Portugal y España dicha tasa se situaba por encima del 9,0 y 7,0, respectivamente, a principios de la década de los años 70 , mientras que dicha cifra ha disminuido progresivamente en todos los países de la UE-15 hasta situarse, en el año 2012, por debajo de 4, presentando España y Portugal tasas similares de nupcialidad en los últimos años.

Tabla II.

Tasa bruta de nupcialidad.

\begin{tabular}{|l|c|c|c|c|}
\hline & $\mathbf{1 9 7 0}$ & $\mathbf{1 9 8 0}$ & $\mathbf{2 0 0 0}$ & $\mathbf{2 0 1 2}$ \\
\hline Grecia & $\mathbf{7 , 7}$ & $\mathbf{6 , 5}$ & 4,5 & 4,5 \\
\hline España & $\mathbf{7 , 3}$ & $\mathbf{5 , 9}$ & $\mathbf{5 , 4}$ & $\mathbf{3 , 5}$ \\
\hline Italia & $\mathbf{7 , 3}$ & 5,7 & 5,0 & 3,5 \\
\hline Portugal & $\mathbf{9 , 4}$ & $\mathbf{7 , 4}$ & $\mathbf{6 , 2}$ & $\mathbf{3 , 3}$ \\
\hline
\end{tabular}

Fuente: Eurostat.

En un contexto en el que las mujeres apenas participaban en el mercado de trabajo y, por tanto, con frecuencia no aportaban renta al hogar, hacía que el matrimonio resultara "rentable" y casarse era una opción más interesante que permanecer soltera
(Martínez-Pastor 2008). Sin embargo, esta tendencia ha cambiado en las últimas décadas al aumentar su participación en el mercado laboral. Además, en la primera década del nuevo siglo se ha producido un rápido aumento de la cohabitación no matrimonial como vía de formación familiar (Domínguez-Folgueras y Castro-Martín 2013).

El retraso en la formación del hogar (tan típica de las sociedades del sur de Europa), la situación del mercado de trabajo local (con elevadas tasas de paro y grandes dificultades para encontrar un trabajo estable) o la escasez de viviendas asequibles (Esping-Andersen 2013), son algunos de los factores que explican que en el último tercio del siglo $X X$ se haya experimentado, en los países industrializados, una considerable reducción de las tasas de fecundidad. En los años 80 países como Portugal o España superaban los dos hijos por mujer; sin embargo, a principios del siglo XXI ninguno de los países de la UE-15 alcanzaba una tasa de fecundidad de dos hijos por mujer. Además, cabe mencionar que, España, junto a Portugal, Grecia e Italia, destaca por ser uno de los países que presenta una de las tasas más bajas, poco más de un hijo por mujer (Tabla III).

Tabla III.

Tasa de fecundidad.

\begin{tabular}{|l|c|c|c|c|}
\hline & $\mathbf{1 9 8 0}$ & $\mathbf{1 9 9 0}$ & $\mathbf{2 0 0 0}$ & $\mathbf{2 0 1 4}$ \\
\hline Grecia & 2,23 & 1,40 & 1,27 & 1,30 \\
\hline España & $\mathbf{2 , 2 0}$ & $\mathbf{1 , 3 6}$ & $\mathbf{1 , 2 3}$ & $\mathbf{1 , 3 2}$ \\
\hline Italia & 1,64 & 1,33 & 1,26 & 1,37 \\
\hline Portugal & $\mathbf{2 , 2 5}$ & $\mathbf{1 , 5 6}$ & $\mathbf{1 , 5 5}$ & $\mathbf{1 , 2 3}$ \\
\hline
\end{tabular}

Fuente: Eurostat.

A comienzos de los años 2000 se observaban algunas señales de recuperación en las tasa de fecundidad en muchos países desarrollados, alcanzándose tasas de fecundidad cercanas al nivel de reemplazo. El aumento de la inmigración y las políticas sociales de apoyo a las familias pudieron haber contribuido a la recuperación (Luci y Thévenon 2012). Sin embargo, el inicio de la crisis económica en el año 2008 trajo consigo una disminución de las tasas de fecundidad en numerosos países y especialmente en los países del sur de Europa, en parte como consecuencia de la reducción de los flujos migratorios y de la formación de parejas (Sobotka et al. 2011).

A las dificultades que tienen las mujeres españolas y portuguesas para integrarse en el mercado laboral, hay que unir otros factores como el reducido nivel de protección social (tanto en gasto público a la familia como en la provisión de cuidados a los niños en sus primeros años de vida) que dificultan la compatibilización laboral y familiar. En España y Portugal el gasto público en familia, medido como porcentaje del PIB a precios corrientes, se sitúa en poco más de un $1 \%$, cifra que queda aún muy lejos de las ci- 
fras de países como Dinamarca o Reino Unido, que alcanzan un porcentaje del $4 \%$. En lo relativo a los servicios de atención a la infancia, la tasa de escolarización de los niños de 0 a 2 años en España y Portugal en el año 2012, en torno al 40\%, son bajas si las comparamos con las de otros países europeos como Dinamarca (67\%), Luxemburgo $(56.3 \%)$ o Países Bajos $(53.9 \%)$ pero, están bastante por encima de los otros dos países que pertenecen al modelo Mediterráneo (Italia, 22.8\%; Grecia, 22.9\%). De hecho, la provisión de centros de educación infantil se ha convertido en el principal estímulo para la participación laboral femenina (Del Boca 2002).

Por tanto, parece que, en las últimas décadas se han producido importantes transformaciones en el ámbito laboral y familiar de las mujeres españolas y portuguesas. En ambos países se ha producido una disminución del número de matrimonios, un descenso en las tasas de fecundidad (aunque con una cierta recuperación a principios del siglo XXI) y un papel importante de la familia como proveedora de servicios dentro del ámbito privado (Ferrera 1996; Bettio y Villa 1998; Naldini 2003; Boeri et al. 2005; Moreno 2008). Sin embargo, si atendemos a las tasas de empleo femenino, a pesar del importante aumento de la participación laboral de las mujeres españolas desde la segunda mitad de los años 80 , Portugal tiene un comportamiento más parecido a los países del centro de Europa que al resto de países sureños (Miller 2004).

\section{MARCO TEÓRICO}

La familia ha atravesado un cúmulo de cambios vertiginosos en las últimas décadas. Cada vez más, las mujeres retrasan la edad del matrimonio y de la primera maternidad, y un número creciente de las parejas en las sociedades occidentales optan voluntariamente por no tener descendencia.

Varios enfoques teóricos ponen énfasis en la maternidad como una decisión racional que recoge las preferencias y las circunstancias vitales de las mujeres. Desde el ámbito socioeconómico, el aumento de la participación laboral de las mujeres, el descenso del número de matrimonios y el incremento en el de parejas sin hijos puede ser explicado desde el marco teórico de la Nueva Economía de la Familia, desarrollado por Gary Becker en 1960. Según esta teoría la unidad familiar distribuye su tiempo entre el mercado de trabajo (que financia la adquisición de bienes de mercado necesarios para producir bienes domésticos) y el trabajo doméstico (donde se incluye el tiempo dedicado a la crianza y la educación de los hijos). Cada miembro de la familia se especializará en la actividad en la que tenga mayor productividad, que viene dada por su stock de capital humano. Si el capital humano adquirido en el sistema educativo aumenta la productividad laboral más fuera que en la producción doméstica, su rentabilidad orienta- rá la asignación del tiempo de las personas hacia el mercado de trabajo en lugar del ámbito doméstico. De forma que las mujeres con mayor nivel educativo participarán más en el mercado de trabajo. Además, según este autor, los padres con mayor nivel educativo parecen valorar más la calidad que la cantidad de hijos, de forma que los mayores ingresos laborales pueden llevarles a sustituir cantidad por calidad de los hijos. Por tanto, varias hipótesis podrían surgir de esta teoría: las mujeres más educadas, aquellas de cohortes más recientes, las que viven en zonas con mayores oportunidades laborales $y$, por tanto, tienen un mayor coste de no participar, tendrán mayor probabilidad de participar en el mercado de trabajo y tener menos hijos.

También los cambios en los patrones de fecundidad en Europa se pueden analizar desde la perspectiva de la Segunda Transición Demográfica (STD) (van de Kaa 1997), que recoge un proceso de reducción de la fecundidad, aumento de emparejamientos distintos al matrimonio, disociación entre el matrimonio y la procreación, entre otras transformaciones sociales y de valores. De hecho, los países correspondientes al arco mediterráneo, como España y Portugal, están viviendo esta Segunda Transición Demográfica de forma especialmente pronunciada. Esta transición demográfica está relacionada con la mayor formación de las mujeres y su mayor vinculación con el mercado de trabajo, así como con nuevos valores y preferencias. Este análisis demográfico no dista mucho del económico ni en supuestos, ni en hipótesis y métodos, si bien pone más énfasis en las preferencias de las mujeres.

Un argumento paralelo desde la sociología explica cómo la elección del estilo de vida que tienen las mujeres ejerce una importante influencia en sus decisiones de maternidad/no maternidad y participación laboral. En la Teoría de la Preferencia Hakim se clasifica a las mujeres en tres grupos según sus aspiraciones y prioridades en relación familia-trabajo (Hakim 2000; 2003a): mujeres centradas en la familia, mujeres centradas en el trabajo y mujeres "adaptativas". Las mujeres más orientadas al mercado laboral pasan más años en el sistema educativo para conseguir mayores logros laborales; es decir, se trata mayoritariamente de mujeres con un mayor nivel educativo. De ahí que, según este enfoque, las mujeres con mayor nivel de estudios pueden ser más propensas a no tener hijos o retrasar más la maternidad ya que están más centradas en el mercado de trabajo en comparación con las mujeres con menor nivel educativo. Sin embargo, son numerosas las críticas que ha recibido este enfoque dado el poco énfasis que le proporciona tanto al propio proceso de formación de las preferencias individuales (que está influido tanto por el entorno como por las circunstancias individuales de cada mujer) como por otros factores que pueden determinar la participación laboral 
de las mujeres (tales como las políticas familiares o la regulación del empleo, que pueden influir en la estrategia elegida por las mujeres para compatibilizar su vida laboral y sus responsabilidades familiares). Así, Warren (2000) y Walters (2005) destacan la necesidad de entender las preferencias en el contexto en que se forman, mientras que Crompton y Lyonette (2005) describen el argumento de Hakim como voluntarista y que no tiene en cuenta que las elecciones de las mujeres permanecen arraigadas en contextos culturares y sociales.

Hakim reconoce que la influencia de las preferencias de las mujeres es más limitada en aquellas sociedades donde el Nuevo Escenario todavía no está completamente desarrollado, como sucede en España (o Portugal), en comparación con otros países como Reino Unido (Hakim 2003b). Los cinco cambios económicos y sociales que definen este Nuevo Escenario son: (a) el control de nacimientos, (b) la legislación sobre la igualdad de oportunidades, (c) la expansión del trabajo de cuello blanco, (d) la creación de empleos para personas que contribuyen con ingresos secundarios y (e) la creciente importancia de las actitudes, valores y preferencias personales en las opciones de estilos de vida. En los países del sur de Europa se produjo una incorporación más tardía de los anticonceptivos modernos, mayores restricciones al aborto, una tardía aplicación de políticas familiares más audaces en materia de conciliación de la vida laboral y familiar que nos acercaran a las que se estaban llevando a cabo en otros países europeos, una menor incidencia de la cohabitación, de los nacimientos fuera del matrimonio o del divorcio. Según los críticos de la teoría de la preferencia de Hakim, la configuración institucional nacional puede influir directamente en las decisiones de las mujeres. En el caso de los países del sur de Europa, la expansión educativa puede explicar que las mujeres esperen hasta tener una posición estable en el mercado de trabajo para tener su primer hijo o incluso decidan no ser madres. Sin embargo, en otros países europeos donde las mujeres presentan elevados niveles educativos, la participación laboral de las mujeres es más elevada y no se retrasa tanto la maternidad (Gutiérrez-Domènech 2008). Por lo tanto, aunque la participación laboral de las mujeres ha aumentado y el modelo del varón-sustentador está desapareciendo en los países sureños; el marco institucional, tanto español como portugués, juega un papel importante a la hora de explicar las bajas tasas de fecundidad en las cohortes recientes de ambos países.

\section{BASE DE DATOS Y METODOLOGÍA}

Los datos utilizados en este artículo proceden de la Encuesta de la Unión Europea sobre Condiciones de Vida (en adelante, EU-SILC), que es un instrumento destinado a la producción de estadísticas armonizadas para los países de la Unión Europea. La
EU-SILC proporciona información comparable y armonizada sobre los siguientes aspectos: ingresos de los hogares privados; pobreza y privación, igualdad de trato del hombre y de la mujer; empleo, actividad y cuidado de niños; jubilaciones, pensiones y situación socioeconómica de las personas de edad; vivienda y desarrollo regional y movimientos migratorios. La población de referencia de la EU-SILC es todos los hogares privados y sus miembros actuales que residan en el territorio en el momento de la recolección de datos. En este trabajo se analizará la muestra española y portuguesa de la EU-SILC (2005-2013).

Con el objetivo de abordar el análisis de las decisiones de participación laboral, convivencia en pareja y de no ser madres estudiaremos un conjunto de mujeres al final de su ciclo reproductivo (entre los 35 y 40 años de edad). Tras excluir las observaciones que no proporcionan toda la información necesaria en el modelo multivariante, la muestra final asciende a 18.143 mujeres, de las cuales aproximadamente el $75 \%$ son mujeres españolas y el $25 \%$ son mujeres portuguesas.

La estrategia empírica más adecuada en este caso consiste en el planteamiento de un modelo probit trivariante y de carácter recursivo, cuyas variables dependientes son (1) participación en el mercado de trabajo, (2) convivencia en pareja y (3) no tener hijos. Cada una de estas variables toma valor 1 cuando se dan dichos eventos y valor 0 en caso contrario. La especificación general del modelo probit trivariante es la siguiente (para una explicación más detallada del modelo, véase Greene 2003):

$$
\begin{aligned}
& y_{i m}{ }^{*}=\beta_{m}, x_{i m}+\varepsilon_{i m}, m=1,2,3(1) \\
& y_{i m}=1 \text { si } y_{i m}{ }^{*}=>0 \text { y } 0 \text { en otro caso (2) }
\end{aligned}
$$

donde $y_{i 1}{ }^{*}, y_{i 2}{ }^{*}$ e $y_{i 3}{ }^{*}$ son tres variables latentes que denotan la propensión de trabajar, la de convivir en pareja y la de no tener hijos, respectivamente; $x_{i m}(m=1,2,3)$ son los vectores de las características observables que afectan a las probabilidades estimadas; $\beta_{m}(m=1,2,3)$ son los vectores de parámetros a estimar; $\varepsilon_{i m}$ son los términos de error. La naturaleza recursiva del modelo la da el hecho de que en cada una de las ecuaciones se incorporan como factores explicativos las variables dependientes en las otras dos. Esta estrategia es preferible a estimar las tres ecuaciones por separado porque es poco probable que las decisiones analizadas sean ciertamente exógenas (independientes) entre sí, esto es, podemos esperar que las tres decisiones estén inter-relacionadas entre sí. Con el objetivo de hacer frente a este posible problema de endogeneidad en el modelo probit trivariante se estiman las tres variables dependientes de manera simultánea, teniendo en cuenta la posibilidad de que haya determinantes inobservados comunes a las tres decisiones. De no considerarse la posible existencia de dichos factores inobservados y las interrelaciones entre las diferentes decisiones, obtendríamos estimaciones sesgadas e ineficientes. 
La especificación de este modelo trivariante tiene en cuenta la potencial correlación entre las diferentes decisiones familiares y laborales. Así vendría recogido en la matriz de varianzas-covarianzas de los términos de error $\left(\varepsilon_{i m}\right)$ identificados en la ecuación (1) incluye correlaciones potencialmente distintas de cero fuera de la diagonal principal. La especificación conjunta de $\varepsilon_{i m}$ sigue una distribución Normal multivariante (DNM):

$$
\left(\varepsilon_{i 1}, \varepsilon_{i 2}, \varepsilon_{i 3}\right)^{\prime} \sim D N M\left(0,\left[\begin{array}{ccc}
1 & \rho_{12} & \rho_{13} \\
\rho_{12} & 1 & \rho_{23} \\
\rho_{13} & \rho_{23} & 1
\end{array}\right]\right)(3)
$$

donde $\rho_{i m}$ es el coeficiente de correlación de $\varepsilon_{j}$ y $\varepsilon_{m}$ para $j \neq m$ (3). Para estimar el modelo probit multivariante se ha utilizado el comando mvprobit de Stata, creado por Cappellari y Jenkins (2003).

En la determinación del modelo las tres ecuaciones tienen una especificación muy parecida: en todas ellas se hace depender cada una de las variables exógenas de vectores $x_{1}, x_{2}$ y $x_{3}$ muy parecidos. Todos ellos se componen de variables que describen tres ámbitos: (a) características sociodemográficas (edad, nivel educativo, país de nacimiento y problemas de salud), (b) una relacionada con la composición familiar (si vive con los padres en ese momento) y (c) una vinculada a características del entorno, en particular, se ha incorporado el grado de urbanización. Adicionalmente, se incorpora como última variable de control el año de la entrevista. Además, en la ecuación de no maternidad se incluye una variable que identifica si la mujer se encuentra en riesgo de pobreza en el momento de la entrevista (en la tabla Al del Apéndice se presenta una descripción de las variables explicativas incluidas en los modelos).

Los valores medios de todas las variables incluidas en el modelo multivariante se muestran en la tabla All del Apéndice. De esta tabla se deduce que las muestras de mujeres españolas y portuguesas son distintas. Una mayor proporción de mujeres portuguesas participan en el mercado laboral, no tienen pareja y no tienen hijos en comparación con las mujeres españolas. Además, las mujeres españolas presentan un nivel educativo más alto y un mayor porcentaje no vive con los padres. Por otro lado, un porcentaje más alto de mujeres españolas están en riesgo de pobreza y residen en zonas muy pobladas. En los resultados del modelo econométrico presentados en el siguiente apartado confirmaremos si las diferencias en el marco institucional o las distintas preferencias de las mujeres españolas y portuguesas, pueden hacer que las características de las mujeres y su entorno influyan de manera diferente en sus decisiones laborales y familiares.

\section{Resultados}

En la Tabla IV se recogen los resultados del modelo probit multivariante. Se observa, por un lado, que la edad biológica no parece influir en la probabilidad de trabajar, en línea con los resultados obtenidos por Gutiérrez-Domènech (2005) y Sheran (2007) que no encontraban una influencia significativa de la edad de la mujer sobre sus decisiones de participación laboral ${ }^{3}$. Por otro lado, la edad sólo resulta negativamente significativa en las decisiones de convivencia en pareja de las mujeres portuguesas más mayores de la muestra. Esto puede ser debido a que el inicio de la primera convivencia en pareja no viene tan determinado por la edad biológica como por la fecha de finalización de los estudios y el inicio de la actividad laboral (Delgado et al. 2009). Sin embargo, cabe destacar que, la edad tiene un impacto diferente en las decisiones de maternidad de las mujeres españolas y portuguesas. Mientras esta variable no resulta significativa para las mujeres portuguesas, un país donde el número de mujeres sin hijos se sitúa en un nivel bajo (Miettinen et al. 2015) y, por tanto, las mujeres han podido tomar sus decisiones de maternidad antes de los 35 años. En el caso de las mujeres españolas conforme avanza su edad disminuye la probabilidad de que éstas no tengan hijos, es decir, la edad tiene un impacto positivo en la decisión de tener un hijo (Del Boca et al. 2005). España es uno de los países donde más se retrasa la primera maternidad (Legazpe 2016).

En cuanto al nivel educativo se observa que, tanto en el caso de las mujeres españolas como en el de las mujeres portuguesas, un mayor número de años en el sistema educativo tiene un impacto positivo en la participación laboral y un efecto negativo sobre la probabilidad de iniciar una convivencia en pareja y tener hijos. Tal y como se desprende de las teorías recogidas en la Sección 3, el capital humano adquirido en el sistema educativo genera mayor productividad en el mercado de trabajo, los rendimientos de esta inversión orientarán el tiempo de las mujeres hacia el mercado de trabajo en lugar de a la producción en el ámbito doméstico. De forma que, las mujeres con mayor nivel educativo tendrán mayor probabilidad de no tener hijos a lo largo de su vida fértil (Kneale y Joshi 2008). El impacto positivo de la educación sobre las decisiones de participación y el efecto negativo en las decisiones de maternidad confirman los resultados obtenidos previamente por Tavora (2012) y Mendes et al. (2006) en Portugal y por Álvarez-Llorente (2002) y Davia y Legazpe (2014) en España.

Respecto al resto de variables sociodemográficas incluidas en los modelos, en la Tabla IV, se observa, en primer lugar, que las mujeres extranjeras que residen en España tienen menos probabilidad de trabajar y ser madres, pero muestran una mayor probabilidad de convivir en pareja. La crisis económica acontecida en España en los últimos años ha afectado de manera importante a la población extranjera, que ha visto reducidas las posibilidades de encontrar un empleo en España (Álvarez et al. 2013). Si bien las oportunidades de empleo son reducidas, las mujeres extranjeras más jóvenes y sin responsabilidades familiares tendrán ma- 
yores posibilidades de ascender en el mercado laboral español (Priegue 2008). Esto unido a que las mujeres inmigrantes se suelen enfrentar a un mayor coste de vida en España que el de su país de procedencia (con una oferta de servicios públicos escasos y de servicios privados con elevado coste que dificultan la conciliación de la vida familiar y laboral) y también encuentran mayores oportunidades educativas y laborales, todo ello aumentará sus costes reales y de oportunidad de tener hijos (Castro-Martín y Rosero-Bixby 2011). En el caso de las mujeres extranjeras que residen en Portugal, parece que, el país de nacimiento no influye a la hora de tomar sus decisiones familiares y laborales. Portugal es uno de los países de la OCDE que presenta una de las tasas de ocupación más altas de las mujeres inmigrantes. El aumento de la tasa de participación de las mujeres portuguesas ha derivado en un aumento de la demanda de trabajadoras inmigrantes en el sector doméstico. Tal es su prevalencia en este país que el trabajo doméstico está regulado por una legislación específica (tipos de contrato, horas de trabajo, vacaciones, etc.), aunque siguen existiendo elevados niveles de informalidad en este sector (Wall y Nunes 2010).

Además, en este país las mujeres inmigrantes que participan en el mercado laboral utilizan servicios de cuidado infantil a tasas similares a las mujeres nativas trabajadoras (Rubin et al. 2008). Por tanto, parece que, en Portugal no existen grandes diferencias entre mujeres nativas e inmigrantes a la hora de la toma de las decisiones laborales y familiares. En segundo lugar, las mujeres que no tienen problemas de salud muestran mayor probabilidad de trabajar (Cai 2010) y convivir en pareja en ambos países. Becker presenta una analogía entre la inversión en salud y en otras formas de capital humano como la salud. Según la teoría del capital humano, tener salud y participar en el mercado de trabajo están positivamente correlacionados. Mientras que gozar de buena salud afecta positivamente a las decisiones de maternidad de las mujeres españolas, dicha variable no resulta significativa a la hora de tomar la decisión de tener un hijo para las mujeres portuguesas. El impacto positivo del estado de salud sobre las decisiones de maternidad, en otros países del sur de Europa, ha sido analizado previamente en Hondroyiannis (2004). Por último, las mujeres que se encuentran en una situación de riesgo de pobreza tienen mayor probabilidad de tener hijos en ambos países (Legazpe 2015). Este resultado confirma que existe una división socio-económica en el comportamiento reproductivo y que la mayor proporción de descendencia numerosa (tres o más hijos) se da entre las parejas socialmente desfavorecidas (Bernardi 2003).

La siguiente variable incorporada en los modelos está relacionada con la composición familiar y, concretamente si la mujer reside con sus padres o suegros en el mismo domicilio en el momento de la entrevista. Si la mujer convive con sus padres en el mismo domicilio disminuye la probabilidad de que ésta conviva en pareja y que tenga hijos. Además, en el caso de las mujeres portuguesas disminuye la probabilidad de estar participando en el mercado laboral. La presencia de una persona adulta dependiente en el hogar tiene un importante impacto negativo en la participación laboral de las mujeres, en línea con los resultados obtenidos por Ettner (1995).

Finalmente, se han incluido en los modelos dos variables adicionales, una vinculada a las características del entorno, concretamente, el grado de urbanización; y otra que recoge los cambios en las decisiones laborales y familiares de las mujeres a lo largo del ciclo económico. Respecto a la primera variable, en la Tabla IV, se observa que en las zonas muy pobladas hay menor probabilidad de que las mujeres convivan en pareja y tengan hijos. El grado de urbanización, y las diferencias urbano-rurales, está asociado a diferentes entornos socio-culturales en términos de valores, preferencias y actitudes hacia las decisiones familiares (Mendes et al. 2006). Mientras que dicha variable sólo afecta a la participación laboral en el caso de las mujeres españolas, que tienen mayor probabilidad de participar en el mercado de trabajo si residen en zonas muy pobladas. Los resultados confirman que las mujeres que residen en zonas con mayores oportunidades laborales, como Madrid o Barcelona, tienen una mayor vinculación al mercado laboral, incluso tienen menor probabilidad de abandonar la actividad laboral ante cambios en sus circunstancias familiares, como el nacimiento de un hijo (Alba y Álvarez-Llorente 2004). En el caso de Portugal, aunque en las últimas décadas ha aumentado la importancia del sector terciario, el sector agrícola sigue teniendo un tamaño considerable. Existe una larga tradición de la participación laboral femenina en la agricultura, ya sea como asalariado o en la agricultura de subsistencia, dado que el flujo migratorio de los hombres durante la década de los 60 convirtió a muchas mujeres en propietarias de los pequeños negocios familiares. Lo que puede explicar que el grado de urbanización no sea significativo a la hora de explicar la participación laboral de las mujeres portuguesas.

En cuanto a la segunda variable, los resultados muestran que la actual crisis económica, que está afectando especialmente a los países sureños de Europa, ha tenido un impacto negativo en la participación laboral de las mujeres y en sus decisiones de iniciar una convivencia en pareja y tener hijos. Durante los años de expansión económica y primeros años de crisis las mujeres españolas tenían mayor probabilidad de participar en el mercado laboral, lo que puede reflejar el efecto del trabajador añadido, mientras que dicha variable no resultaba significativa en el caso de las mujeres portuguesas, que siempre han mostrado elevadas tasas de participación laboral; por el contrario, en los años de crisis económica las mujeres portuguesas han reducido la probabilidad de participar en el mercado de trabajo. 
Tabla IV.

Modelos probit multivariante

\begin{tabular}{|c|c|c|c|c|c|c|c|}
\hline & & \multicolumn{3}{|c|}{ España } & \multicolumn{3}{|c|}{ Portugal } \\
\hline & & Trabajo & Pareja & Sin hijos & Trabajo & Pareja & Sin hijos \\
\hline \multirow{5}{*}{$\begin{array}{l}\text { Edad } \\
\text { (ref. } 35 \text { años) }\end{array}$} & 36 años & $\begin{array}{c}0,040 \\
(0,040)\end{array}$ & $\begin{array}{c}0,001 \\
(0,048)\end{array}$ & $\begin{array}{l}-0,054 \\
(0,043)\end{array}$ & $\begin{array}{c}0,012 \\
(0,077)\end{array}$ & $\begin{array}{l}-0,039 \\
(0,089)\end{array}$ & $\begin{array}{l}-0,012 \\
(0,088)\end{array}$ \\
\hline & 37 años & $\begin{array}{l}-0,010 \\
(0,040)\end{array}$ & $\begin{array}{c}0,026 \\
(0,049)\end{array}$ & $\begin{array}{c}-0,167^{* \star *} \\
(0,043)\end{array}$ & $\begin{array}{c}0,044 \\
(0,076)\end{array}$ & $\begin{array}{l}-0,074 \\
(0,087)\end{array}$ & $\begin{array}{l}-0,015 \\
(0,086)\end{array}$ \\
\hline & 38 años & $\begin{array}{l}-0,050 \\
(0,039)\end{array}$ & $\begin{array}{c}0,063 \\
(0,048)\end{array}$ & $\begin{array}{c}-0,265^{\star \star *} \\
(0,044)\end{array}$ & $\begin{array}{c}0,116 \\
(0,076)\end{array}$ & $\begin{array}{l}-0,145^{\star} \\
(0,086)\end{array}$ & $\begin{array}{l}-0,006 \\
(0,086)\end{array}$ \\
\hline & 39 años & $\begin{array}{l}-0,031 \\
(0,039)\end{array}$ & $\begin{array}{c}0,054 \\
(0,048)\end{array}$ & $\begin{array}{c}-0,360^{\star * *} \\
(0,044)\end{array}$ & $\begin{array}{c}0,077 \\
(0,075)\end{array}$ & $\begin{array}{l}-0,120 \\
(0,086) \\
\end{array}$ & $\begin{array}{c}-0,114 \\
(0,087) \\
\end{array}$ \\
\hline & 40 años & $\begin{array}{l}-0,019 \\
(0,039)\end{array}$ & $\begin{array}{c}0,073 \\
(0,048)\end{array}$ & $\begin{array}{c}-0,332^{* * *} \\
(0,044)\end{array}$ & $\begin{array}{c}0,091 \\
(0,074)\end{array}$ & $\begin{array}{c}-0,169^{* *} \\
(0,085)\end{array}$ & $\begin{array}{l}-0,087 \\
(0,086) \\
\end{array}$ \\
\hline \multirow{3}{*}{$\begin{array}{l}\text { Nivel educativo } \\
\text { (ref. Educación secundaria obligatoria) }\end{array}$} & $\begin{array}{l}\text { Educación } \\
\text { secundaria } \\
\text { superior }\end{array}$ & $\begin{array}{c}0,426^{* * *} \\
(0,030)\end{array}$ & $\begin{array}{l}-0,056 \\
(0,038)\end{array}$ & $\begin{array}{c}0,231^{* * *} \\
(0,036)\end{array}$ & $\begin{array}{c}0,541^{* * *} \\
(0,057)\end{array}$ & $\begin{array}{l}-0,145^{\star *} \\
(0,060)\end{array}$ & $\begin{array}{l}0,120^{*} \\
(0,065)\end{array}$ \\
\hline & \begin{tabular}{|l}
$\begin{array}{l}\text { Estudios } \\
\text { superiores }\end{array}$ \\
\end{tabular} & $\begin{array}{c}0,752^{* * *} \\
(0,027)\end{array}$ & $\begin{array}{c}-0,131^{\star * *} \\
(0,033)\end{array}$ & $\begin{array}{c}0,434^{\star * *} \\
(0,032)\end{array}$ & $\begin{array}{c}1,006^{* * *} \\
(0,069)\end{array}$ & $\begin{array}{c}-0,146^{* *} \\
(0,064)\end{array}$ & $\begin{array}{c}0,475^{* * *} \\
(0,064)\end{array}$ \\
\hline & No contesta & $\begin{array}{c}-0,944^{\star * *} \\
(0,131)\end{array}$ & $\begin{array}{c}-0,092 \\
(0,136)\end{array}$ & $\begin{array}{l}-0,081 \\
(0,131)\end{array}$ & $\begin{array}{l}- \\
- \\
\end{array}$ & $\begin{array}{l}- \\
- \\
\end{array}$ & $\begin{array}{l}- \\
- \\
\end{array}$ \\
\hline $\begin{array}{l}\text { País de nacimiento } \\
\text { (ref. Mismo país que residencia) }\end{array}$ & $\begin{array}{l}\text { Otro país distinto } \\
\text { al de residencia }\end{array}$ & $\begin{array}{l}-0,120^{\star * *} \\
(0,036)\end{array}$ & $\begin{array}{l}0,169^{* * *} \\
(0,045)\end{array}$ & $\begin{array}{l}0,122^{* * *} \\
(0,041)\end{array}$ & $\begin{array}{l}-0,103 \\
(0,073) \\
\end{array}$ & $\begin{array}{c}0,034 \\
(0,079)\end{array}$ & $\begin{array}{l}-0,051 \\
(0,081)\end{array}$ \\
\hline \multirow{2}{*}{$\begin{array}{l}\text { Problemas de salud } \\
\text { (ref. Sí) }\end{array}$} & $\begin{array}{l}\text { No tiene } \\
\text { problemas } \\
\text { de salud }\end{array}$ & $\begin{array}{l}0,325^{\star \star *} \\
(0,033)\end{array}$ & $\begin{array}{l}0,197^{* * *} \\
(0,039)\end{array}$ & $\begin{array}{c}-0,150^{* * *} \\
(0,037)\end{array}$ & $\begin{array}{l}0,414^{\star * *} \\
(0,057)\end{array}$ & $\begin{array}{l}0,240^{* \star *} \\
(0,067)\end{array}$ & $\begin{array}{l}-0,031 \\
(0,071)\end{array}$ \\
\hline & No contesta & $\begin{array}{c}2,680^{* * *} \\
(0,459)\end{array}$ & $\begin{array}{c}0,298 \\
(0,379) \\
\end{array}$ & $\begin{array}{l}0,776^{* *} \\
(0,335)\end{array}$ & $\begin{array}{l}-0,073 \\
(0,799) \\
\end{array}$ & $\begin{array}{c}0,993 \\
(1,137) \\
\end{array}$ & $\begin{array}{c}-1,676^{\star *} \\
(0,742)\end{array}$ \\
\hline $\begin{array}{l}\text { Riesgo de pobreza } \\
\text { (ref. No) }\end{array}$ & $\begin{array}{l}\begin{array}{l}\text { Sí, en riesgo de } \\
\text { pobreza }\end{array} \\
\end{array}$ & - & - & $\begin{array}{c}-0,352^{* * *} \\
(0,037)\end{array}$ & - & - & $\begin{array}{c}-0.349^{* * *} \\
(0.074)\end{array}$ \\
\hline $\begin{array}{l}\text { Vive con los padres } \\
\text { (ref. No) }\end{array}$ & $\begin{array}{l}\text { Sí, vive con los } \\
\text { padres }\end{array}$ & $\begin{array}{l}-0,015 \\
(0,032)\end{array}$ & $\begin{array}{c}-1,869^{\star \star *} \\
(0,034)\end{array}$ & $\begin{array}{c}0,891^{* * *} \\
(0,032)\end{array}$ & $\begin{array}{c}-0,241^{* * *} \\
(0,053)\end{array}$ & $\begin{array}{c}-1,326^{\star \star *} \\
(0,056)\end{array}$ & $\begin{array}{c}0.777^{* * *} \\
(0.057)\end{array}$ \\
\hline \multirow{2}{*}{$\begin{array}{l}\text { Grado de urbanización } \\
\text { (ref. Zona muy poblada) }\end{array}$} & Zona media & $\begin{array}{c}-0,152^{* * *} \\
(0,029)\end{array}$ & $\begin{array}{c}0,237^{* * *} \\
(0,035)\end{array}$ & $\begin{array}{c}-0,147^{* * *} \\
(0,033)\end{array}$ & $\begin{array}{c}0,050 \\
(0,051)\end{array}$ & $\begin{array}{c}0,220^{\star * *} \\
(0,056)\end{array}$ & $\begin{array}{c}-0.084 \\
(0.058)\end{array}$ \\
\hline & \begin{tabular}{|l|}
$\begin{array}{l}\text { Zona poco } \\
\text { poblada }\end{array}$ \\
\end{tabular} & $\begin{array}{c}-0,171^{* * *} \\
(0,027)\end{array}$ & $\begin{array}{c}0,340^{* * *} \\
(0,034)\end{array}$ & $\begin{array}{c}-0,150^{* * *} \\
(0,031)\end{array}$ & $\begin{array}{c}0,035 \\
(0,053) \\
\end{array}$ & $\begin{array}{c}0,302^{* * *} \\
(0,060)\end{array}$ & $\begin{array}{c}-0.214^{* * *} \\
(0.063)\end{array}$ \\
\hline \multirow{8}{*}{$\begin{array}{l}\text { Año } \\
\text { (ref. Año 2005) }\end{array}$} & Año 2006 & $\begin{array}{l}0,095^{\star *} \\
(0,046)\end{array}$ & $\begin{array}{c}0,011 \\
(0,058)\end{array}$ & $\begin{array}{c}0,044 \\
(0,054)\end{array}$ & $\begin{array}{c}0,034 \\
(0,092)\end{array}$ & $\begin{array}{l}-0,059 \\
(0,105)\end{array}$ & $\begin{array}{c}0.023 \\
(0.113)\end{array}$ \\
\hline & Año 2007 & $\begin{array}{c}0,132^{* * *} \\
(0,047)\end{array}$ & $\begin{array}{c}0,016 \\
(0,059)\end{array}$ & $\begin{array}{l}0,090^{*} \\
(0,054)\end{array}$ & $\begin{array}{l}-0,080 \\
(0,092) \\
\end{array}$ & $\begin{array}{l}-0,031 \\
(0,107) \\
\end{array}$ & $\begin{array}{c}0.095 \\
(0.113) \\
\end{array}$ \\
\hline & Año 2008 & $\begin{array}{c}0,198^{* * *} \\
(0,046)\end{array}$ & $\begin{array}{c}-0,053 \\
(0,058)\end{array}$ & $\begin{array}{c}0,077 \\
(0,053) \\
\end{array}$ & $\begin{array}{c}0,013 \\
(0,096) \\
\end{array}$ & $\begin{array}{l}-0,137 \\
(0,108) \\
\end{array}$ & $\begin{array}{c}0.109 \\
(0.116) \\
\end{array}$ \\
\hline & Año 2009 & $\begin{array}{c}0,127^{* * *} \\
(0,046)\end{array}$ & $\begin{array}{l}-0,104^{*} \\
(0,057)\end{array}$ & $\begin{array}{c}0,152^{* * *} \\
(0,053)\end{array}$ & $\begin{array}{l}-0,017 \\
(0,094) \\
\end{array}$ & $\begin{array}{c}-6,760 \\
-93,088 \\
\end{array}$ & $\begin{array}{c}2.821^{* \star *} \\
(0.120)\end{array}$ \\
\hline & Año 2010 & $\begin{array}{c}0,132^{\star * *} \\
(0,046) \\
\end{array}$ & $\begin{array}{c}-0,115^{\star *} \\
(0,057)\end{array}$ & $\begin{array}{l}0,110^{* *} \\
(0,053)\end{array}$ & $\begin{array}{l}-0,126 \\
(0,091) \\
\end{array}$ & $\begin{array}{c}-0,315^{\star * *} \\
(0,101)\end{array}$ & $\begin{array}{c}0.109 \\
(0.110) \\
\end{array}$ \\
\hline & Año 2011 & $\begin{array}{c}0,076 \\
(0,048)\end{array}$ & $\begin{array}{c}-0,182^{\star * *} \\
(0,058)\end{array}$ & $\begin{array}{l}0,113^{* *} \\
(0,054)\end{array}$ & $\begin{array}{l}-0,089 \\
(0,090)\end{array}$ & $\begin{array}{c}-0,325^{\star \star *} \\
(0,099)\end{array}$ & $\begin{array}{l}0.258^{* *} \\
(0.105)\end{array}$ \\
\hline & Año 2012 & $\begin{array}{l}-0,058 \\
(0,048)\end{array}$ & $\begin{array}{c}-0,158^{* * *} \\
(0,059)\end{array}$ & $\begin{array}{l}0,092^{*} \\
(0,055)\end{array}$ & $\begin{array}{c}-0,186^{\star *} \\
(0,086)\end{array}$ & $\begin{array}{c}-0,378^{* * *} \\
(0,095)\end{array}$ & $\begin{array}{c}0.280^{* * *} \\
(0.101)\end{array}$ \\
\hline & Año 2013 & $\begin{array}{l}-0,059 \\
(0,048)\end{array}$ & $\begin{array}{c}-0,208^{* * *} \\
(0,059)\end{array}$ & $\begin{array}{c}0,174^{* * *} \\
(0,055)\end{array}$ & $\begin{array}{c}-0,297^{* * *} \\
(0,084)\end{array}$ & $\begin{array}{c}-0,454^{* * *} \\
(0,093)\end{array}$ & $\begin{array}{c}0.291^{* * *} \\
(0.099)\end{array}$ \\
\hline \multicolumn{2}{|l|}{ Constante } & $\begin{array}{c}-0,278^{* * *} \\
(0,054)\end{array}$ & $\begin{array}{c}1,025^{\star \star \star} \\
(0,066)\end{array}$ & $\begin{array}{c}-0,874^{* * *} \\
(0,062)\end{array}$ & $\begin{array}{c}0,163 \\
(0,102)\end{array}$ & $\begin{array}{c}1,157^{* * *} \\
(0,119)\end{array}$ & $\begin{array}{c}-1,343^{* * *} \\
(0,126)\end{array}$ \\
\hline \multicolumn{2}{|l|}{ Rho21 } & \multicolumn{3}{|c|}{$-0,181^{* \star *}(0,016)$} & \multicolumn{3}{|c|}{$0,024(0,029)$} \\
\hline \multicolumn{2}{|l|}{ Rho23 } & \multicolumn{3}{|c|}{$-0,513^{\star * *}(0,019)$} & \multicolumn{3}{|c|}{$-0,452^{* * *}(0,035)$} \\
\hline \multicolumn{2}{|l|}{ Rho31 } & \multicolumn{3}{|c|}{$0,169^{\star \star \star}(0,016)$} & \multicolumn{3}{|c|}{$0,007(0,031)$} \\
\hline \multicolumn{2}{|l|}{ Log Likelihood } & \multicolumn{3}{|c|}{-19526} & \multicolumn{3}{|c|}{-5636} \\
\hline \multicolumn{2}{|l|}{ Chi 2} & \multicolumn{3}{|c|}{5015} & \multicolumn{3}{|c|}{1938} \\
\hline \multicolumn{2}{|l|}{ Número de observaciones } & \multicolumn{3}{|c|}{13570} & \multicolumn{3}{|c|}{4573} \\
\hline
\end{tabular}

Fuente: Encuesta de la Unión Europea sobre Condiciones de Vida (EU-SILC). 
Por último, se analiza la existencia de correlación de los residuos a través de la prueba sobre la hipótesis nula de que el coeficiente de correlación es nulo $(\rho=0)$. En el caso de la muestra de mujeres españolas el test resulta ser significativo y, por tanto, se puede afirmar que existe correlación entre las tres regresiones. El signo negativo del athrho (la aproximación de $\rho$ que ofrece el paquete estadístico utilizado) confirma que las decisiones de participación laboral y convivencia y las decisiones de pareja y de no ser madres están negativamente correlacionadas; mientras que las decisiones de trabajo y de no ser madres están positivamente correlacionadas. En el caso de las mujeres portuguesas sólo se confirma una relación negativa entre las decisiones de convivencia y de no ser madres; mientras que no hay una relación significativa entre las decisiones de participación laboral y las decisiones familiares.

\section{CONCLUSIONES}

En el presente trabajo se ha explotado la Encuesta de la Unión Europea sobre Condiciones de Vida (EU-SILC) para estudiar los factores determinantes de las decisiones laborales, de convivencia en pareja y de no ser madres de las mujeres trabajadoras en España y Portugal y analizar si existen diferencias en ambos países. Para ello se ha estimado un modelo probit trivariante que ha permitido incorporar factores que afectan simultáneamente a las tres decisiones.

Los resultados muestran que la edad, el país de nacimiento, el estado de salud y el año de la entrevista tienen un impacto distinto en las decisiones laborales y familiares de las mujeres españolas y portuguesas. En el caso de las mujeres portuguesas las decisiones de maternidad no vienen determinadas, al contrario que para las mujeres españolas, por variables como la edad, la nacionalidad o el estado de salud. Mientras que sus decisiones de participación laboral sí se han visto afectadas por la crisis económica. Además, cabe destacar, que se ha confirmado que existe una correlación entre las tres decisiones (participación laboral, convivencia en pareja y no-maternidad) en las mujeres españolas, mientras que para las mujeres portuguesas sólo existe una relación significativa entre las decisiones de convivencia en pareja y no-maternidad. De ahí que las mujeres portuguesas presentan tasas de participación más elevadas independientemente de sus decisiones familiares.

La importancia del nivel educativo en las decisiones familiares y laborales se ha confirmado en ambas muestras, de manera que el nivel educativo alcanzado por las mujeres tiene un importante impacto positivo en las decisiones de participación laboral. La integración de las mujeres en el mercado de trabajo es una vía fundamental para garantizar un desarrollo económico sostenido y asegurar el mantenimiento del Estado de Bienestar. El aumento en el número de trabajadoras, y por tanto, del número de cotizantes al sistema de la Seguridad Social permite incrementar el número de recursos destinados a sostenerlo. Pero también hay que destacar que la mayor participación laboral de las mujeres parece haber afectado de manera negativa a la fecundidad en algunos países europeos, y muy especialmente en la población española y portuguesa, lo que se ha traducido en una nueva estructura de la pirámide poblacional, que puede tener importantes consecuencias económicas y sociales para el futuro.

El cambio en las decisiones laborales y familiares no sólo atiende a las preferencias y actitudes de las mujeres, sino también a otros factores del entorno como las políticas familiares o la regulación del empleo, que han influido en que algunos países del sur de Europa alcancen tasas de fecundidad bajas pero no presenten tasas de empleo femenino comparables a las de los países del norte de Europa. En el caso español y portugués, la incorporación de la mujer al mercado de trabajo, se ha visto afectada por problemas de compatibilización de la vida familiar y laboral dado el poco apoyo institucional a las mujeres con hijos, que queda reflejado en un reducido gasto público en familia y en la falta de una oferta pública más amplia de servicios de atención al cuidado de menores. Además, existe una peculiar estructura del mercado laboral, con una escasa disponibilidad de empleos a tiempo parcial en condiciones aceptables. Dada la rigidez que presentan los mercados laborales de los países del sur de Europa, cabe mencionar que, en estos países el empleo flexible se identifica con empleos pertenecientes a la economía sumergida (trabajo doméstico, trabajo denominado como ayuda familiar y el trabajo estacional), que son ocupados mayoritariamente por mujeres, y el cual no es contabilizado en las estadísticas oficiales (Moreno 2007), lo que podría incidir en las tasas reales de empleo femenino en estos países.

En cualquier caso, la realidad evidencia la necesidad de que en ambos países se realicen políticas familiares más audaces encaminadas a aumentar la participación laboral de las mujeres, lo que supondrá un impacto positivo para el desarrollo socio-económico de los países, y que a la vez potencien el aumento de las tasas de natalidad, y permitan el mantenimiento del actual Estado de Bienestar.

\section{NOTAS}

1. En Portugal el salario mínimo es de 589 euros y en España, de 655,20 euros. Desde 2009 hasta 2016, en España el salario mínimo se ha incrementado 31 euros.

2. Tanto por mil de matrimonios.

3. Las decisiones laborales de las mujeres están afectadas por los condicionantes estructurales del mercado

trabajo local, por tanto, una mujer puede estar participando en el mercado de trabajo, bien mediante el acceso a un empleo o mediante la búsqueda del mismo. En este trabajo, nos referimos a su participación como asalariada o trabajadora por cuenta propia. 


\section{REFERENCIAS BibLIOGRÁfICAS}

Alba, A. y G. Álvarez-Llorente. 2004. "Actividad laboral de la mujer en torno al nacimiento de un hijo." Investigaciones Económicas 28: 429-460.

Álvarez, C., M.A. Davia y N. Legazpe. 2013. "Impacto laboral de la crisis económica: privación de empleo y precariedad." Papeles de Economía Española 135: 83-98.

Álvarez-Llorente, G. 2002. "Decisiones de fecundidad y participación laboral de la mujer en España." Investigaciones Económicas 26: 187-218.

Baizán, P., A. Arnstein y B. Francesco. 2003. "Cohabitation, marriage, and first birth: The interrelationship of formation events in Spain." European Journal of Population 19: 147-169. https://doi.org/10.1023/A:1023343001627

Becker, G.S. 1964. Human Capital. Nueva York: Columbia University Press.

Becker, G.S. 1960. "An economic analysis of fertility". Pp. 209-240 en Demographic and Economic Change in Developed Countries. Princeton, NJ: Princeton University Press.

Bernardi, F. 2003. "El déficit de natalidad en España: Análisis y propuestas para la intervención pública." European Sociological Review, 19(1).

Bettio, F. y P. Villa. 1998. "A Mediterranean perspective on the breakdown of the relationship between participation and fertility." Cambridge Journal of Economics 22: 137-171. https://doi.org/10.1093/oxfordjournals.cje. a013708

Boeri, T., D. Del Boca y C.H. Pissarides. 2005. Women at work: An economic perspective. Oxford: Oxford University Press.

Borra, C. 2010. "Childcare cost and Spanish mother's labour participation." Hacienda Pública Española 194: 9-40.

Cai, L. 2010. "The relationship between health and labour force participation: Evidence from a panel data simultaneous equation model." Labour Economics 17: 77-90. https:// doi.org/10.1016/j.labeco.2009.04.001

Cappellari, L. y S.P. Jenkins. 2003. "Multivariate probit regression using simulated maximum likelihood." The Stata Journal 3: 278-294.

Castro-Martín, T. y L. Rosero-Bixby. 2011. "Maternidades y fronteras. La fecundidad de las mujeres inmigrantes en España." Revista Internacional de Sociología 69: 105-138. https://doi.org/10.3989/ris.2011.iM1.388

Crompton, R. y C. Lyonette. 2005. "The new gender essentialism - domestic and family 'choices' and their relation to attitudes', The British Journal of Sociology 56(4): 601620. https://doi.org/10.1111/j.1468-4446.2005.00085.x

Davia, M.A. y N. Legazpe. 2014. "Determinants of employment decisions after the first child birth in Spain." Journal of Family and Economic Issues 35: 214-227. https://doi. org/10.1007/s10834-013-9360-5

Del Boca, D. 2002. "The effect of child care and part time opportunities on participation and fertility decisions in Italy." Journal of Population Economics 15: 549-573. https://doi.org/10.1007/s001480100089

Del Boca, D., S. Pascua y C. Pronzato. 2005. "Fertility and employment in Italy, France and the UK." Labour 19: 1-77.

Delgado, M., F. López y L. Barrios. 2006. "Déficit de fecundidad en España: factores demográficos que operan sobre una tasa muy inferior al nivel de reemplazo." Revista Española de Investigaciones Sociológicas 115: 197-222. https://doi.org/10.2307/40184771

Delgado, M., F. Zamora, L. Barrios, N. Cámara, I. Alberdi y A.
De Rose. 2009). Fecundidad y trayectoria laboral de las mujeres en España. Madrid: Instituto de la Mujer.

Domínguez-Folgueras, M. y T. Castro-Martín. 2013. "Cohabitation in Spain: No longer a marginal path to family formation." Journal of Marriage and Family 75: 422-437. https://doi.org/10.1111/jomf.12013

Esping-Andersen, G. (ed.). 2013. El déficit de natalidad en Europa: La singularidad del caso español. Barcelona: Obra Social "La Caixa".

Ettner, S.L. 1995. "The impact of "parent care" on female labor supply decisions." Demography 32: 63-80. https://doi. org/10.2307/2061897

Ferrera, M. 1996. "The southern model of welfare in social Europe." Journal of European Social Policy 6(1): 17-37. https://doi.org/10.1177/095892879600600102

Garrido, L. 1993. "Las dos biografías de la mujer en España." Madrid: Instituto de la Mujer.

González, E. y M. Segales. 2013. "Women, gender equality and the economic crisis in Spain." Pp. 228-247 en Women and austerity: The economic crisis and the future for gender equality, editado por M. Karamessini y J. Rubery. New York: Routledge.

Greene, W.H. 2003. Econometric analysis. Pearson Education India.

Guerreiro, M.D.D., A. Torres y C. Lobo. 2007. "Famílias em mudança: configurações, valores e processos de recomposição". Pp. 7-37 en Quotidiano e Qualidade de Vida ( $1^{\mathrm{a}}$ ed.). Lisboa: Celta editora.

Gutiérrez-Domènech, M. 2005. "Employment transitions after motherhood in Spain." Labour 19: 123-148. https://doi. org/10.1111/j.1467-9914.2005.00313.x

Gutiérrez-Domènech, M. 2008. "The impact of the labour market on the timing of marriage and births in Spain." Journal of Population Economics 21: 83-110. https://doi. org/10.1007/s00148-005-0041-z

Hakim, C. 2000. Work-lifestyle choices in the 21st century: Preference theory. Oxford: Oxford University Press.

Hakim, C. 2003a. "A new approach to explaining fertility patterns: Preference theory." Population and Development Review 29: 349-374. https://doi.org/10.1111/ j.1728-4457.2003.00349.x

Hakim, C. 2003b. Models of the family in modern societies: Ideals and realities. Burlington, VT: Ashgate Pub Ltd.

Hondroyiannis, G. 2004. "Modeling household fertility decisions in Greece." The Social Science Journal, 41: 477483. https://doi.org/10.1016/j.soscij.2004.04.003

Kneale, D. y H. Joshi. 2008. "Postponement and childlessness: evidence from two British cohorts." Demographic Research 19: 1935-1968. https://doi.org/10.4054/ DemRes.2008.19.58

Legazpe, N. 2016. "Decisiones de formación de la pareja y maternidad en las mujeres españolas". Revista de Economía Aplicada 72: 23-45.

Legazpe, N. 2015. "Mujer, trabajo y familia en España." El Trimestre Económico 82: 873-896. https://doi. org/10.20430/ete.v82i328.187

Luci, A. y O. Thévenon. 2012. "The impact of family policy packages on fertility trends in developed countries". European Journal of Population 29(4): 387-416. https:// doi.org/10.1007/s10680-013-9295-4

Martínez Pastor, J.I. 2008. "Cada vez menos y más tarde: un análisis de la nupcialidad masculina durante los últimos treinta años en España." REIS: Revista Española de Investigaciones Sociológicas 123: 59-86. https:// doi.org/10.2307/40184893 
Mendes, M.F., C. Rego y A. Caleiro. 2006. "Educação e Fecundidade em Portugal: as diferenças nos níveis de educação influenciam as taxas de fecundidade?." Actas do XII Congresso da APDR, 15-17 Setembro 2006, Viseu, Portugal.

Miettinen, A., A. Rotkirch, I. Szalma, A. Donno y M. L. Tanturri. 2015. "Increasing childlessness in Europe: Time trends and country differences". Families and Societies. Working Paper Series 3.

Miller L.M. 2004. "Participación laboral femenina y Estados de bienestar." Revista Española de Investigaciones Sociológicas 108: 49-74.

Moreno, A. 2007. Familia y empleo de la mujer en los regímenes de bienestar del sur de Europa. Incidencia de las políticas familiares y laborales. Madrid: CIS.

Moreno, A. 2008. "El reducido empleo femenino en los Estados del bienestar del sur de Europa. Un análisis comparado." Revista Internacional de Sociología 66: 129-162.

Naldini, M. 2003. The family in the Mediterranean welfare states. London: Frank Cass.

Priegue, D. 2008. Familia, educación e inmigración. Un programa de intervención pedagógica. Santiago de Compostela: Universidad Santiago de Compostela.

Rubin, J., M. S. Rendall, L. Rabinovich, F. Tsang, C. van Oranje-Nassau y B. Janta. 2008. Migrant women in the European labour force. Cambridge: Rand Europe.

Ruivo, M., M. do Pilar González y J.M. Varejão. 1998. "Why is part-time work so low in Portugal and Spain." Pp. 199-213 en Part-time prospects. An international comparison of part-time work in Europe, North America and the Pacific Rim, editado por J. O'Reilly y C. Fagan. Londres: Routledge.
Sheran, M. 2007. "The career and family choices of women: A dynamic analysis of labor force participation, schooling, marriage, and fertility decisions." Review of Economic Dynamics 10: 367-399. https://doi.org/10.1016/j. red.2006.11.004

Schultz, T.W. 1960. "Capital formation by education." The Journal of Political Economy 68(6): 571-583. https:// doi.org/10.1086/258393

Sobotka, T., V. Skirbekk y D. Philipov. 2011. "Economic recession and fertility in the developed world." Population and Development Review 37: 267-306. https://doi. org/10.1111/j.1728-4457.2011.00411.x

Tavora, I. 2012. "Understanding the high rates of employment among low-educated women in Portugal: A comparatively oriented case study." Gender, Work \& Organization 19: 93-118. https://doi.org/10.1111/j.14680432.2010.00489.x

Van de Kaa, D.J. 1997. "Options and sequences: Europe's demographic patterns." Journal of the Australian Population Association 14: 1-29. https://doi.org/10.1007/ BF03029484

Wall, K. y C. Nunes. 2010. "Immigration, welfare and care in Portugal: Mapping the new plurality of female migration trajectories." Social Policy and Society 9: 397-408. https://doi.org/10.1017/S1474746410000114

Walters, S. 2005. "Making the best of a bad job? Female part-timers' orientations and attitudes to work." Gender, Work and Organization 12: 193-216. https://doi. org/10.1111/j.1468-0432.2005.00270.x

Warren, T. 2000. "Women in low status part-time jobs: A class and gender analysis." Sociological Research Online 4. https://doi.org/10.5153/sro.328

\section{APÉNDICE}

Tabla Al.

Descripción de las variables explicativas.

\begin{tabular}{|l|l|}
\hline Variable & Descripción \\
\hline Edad & $\begin{array}{l}\text { La edad ha sido introducida a través de seis variables ficticias que indican si la mujer pertenece o no a } \\
\text { cada una de las siguientes edades: } 35 \text { años (omitida), 36 años, } 37 \text { años, 38 años, } 39 \text { años y } 40 \text { años. }\end{array}$ \\
\hline Nivel educativo & $\begin{array}{l}\text { La educación de la mujer ha sido incluida a través de cuatro variables ficticias: educación secundaria } \\
\text { obligatoria (omitida), educación secundaria superior, estudios superiores y no contesta. }\end{array}$ \\
\hline País de nacimiento & $\begin{array}{l}\text { Se ha introducido a través de dos variables ficticias: mismo país que residencia (omitida) y otro } \\
\text { país distinto al de residencia. }\end{array}$ \\
\hline Problemas de salud & $\begin{array}{l}\text { Se ha introducido a través de tres variables ficticias: sí tiene problemas de salud (omitida), no } \\
\text { tiene problemas de salud y no contesta. }\end{array}$ \\
\hline Riesgo de pobreza & Esta variable indica si la mujer está o no (omitida) en riesgo de pobreza. \\
\hline Vive con los padres & Se ha introducido a través de una variable dicotómica que indica si la mujer convive con los padres. \\
\hline Grado de urbanización & $\begin{array}{l}\text { Se ha introducido a través de tres variables ficticias: zona muy poblada (omitida), zona media y } \\
\text { zona poco poblada. }\end{array}$ \\
\hline Año & $\begin{array}{l}\text { El año se ha incluido a través de nueve variables ficticias: año 2005, año 2006, año 2007, año 2008, } \\
\text { año 2009, año 2010, año 2011, año 2012 y año 2013. }\end{array}$ \\
\hline
\end{tabular}


Tabla All.

Valores medios de las variables utilizadas en los modelos probit multiviariante.

\begin{tabular}{|c|c|c|c|c|}
\hline & & Total & España & Portugal \\
\hline \multirow{3}{*}{$\begin{array}{l}\text { Variables } \\
\text { dependientes }\end{array}$} & Trabajo & 66,35 & 63,23 & 75,62 \\
\hline & Pareja & 77,79 & 80,01 & 71,18 \\
\hline & Sin hijos & 21,24 & 20,94 & 22,15 \\
\hline \multirow{2}{*}{ País } & España & 74,79 & 100 & - \\
\hline & Portugal & 25,21 & - & 100 \\
\hline \multirow{6}{*}{ Edad } & 35 años & 15,60 & 15,97 & 14,50 \\
\hline & 36 años & 16,12 & 16,30 & 15,57 \\
\hline & 37 años & 16,38 & 16,32 & 16,55 \\
\hline & 38 años & 17,02 & 16,99 & 17,12 \\
\hline & 39 años & 17,33 & 17,21 & 17,71 \\
\hline & 40 años & 17,55 & 17,21 & 18,54 \\
\hline \multirow{4}{*}{ Nivel educativo } & Educación secundaria obligatoria & 41,17 & 35,04 & 59,35 \\
\hline & Educación secundaria superior & 22,32 & 22,84 & 20,77 \\
\hline & Estudios superiores & 35,60 & 40,90 & 19,88 \\
\hline & No contesta & 0,91 & 1,22 & - \\
\hline \multirow{2}{*}{ País de nacimiento } & Mismo país que residencia & 88,95 & 88,64 & 89,85 \\
\hline & Otro país distinto al de residencia & 11,05 & 11,36 & 10,15 \\
\hline \multirow{3}{*}{ Problemas de salud } & Sí, tiene problemas de salud & 13,45 & 13,23 & 14,10 \\
\hline & No tiene problemas de salud & 86,44 & 86,64 & 85,83 \\
\hline & No contesta & 0,12 & 0,13 & 0,07 \\
\hline \multirow{2}{*}{ Riesgo de pobreza } & No en riesgo de pobreza & 79,80 & 79,26 & 81,41 \\
\hline & Sí, en riesgo de pobreza & 20,20 & 20,74 & 18,59 \\
\hline \multirow{2}{*}{ Vive con los padres } & No vive con los padres & 84,46 & 85,28 & 82,02 \\
\hline & Sí, vive con los padres & 15,54 & 14,72 & 17,98 \\
\hline \multirow{3}{*}{ Grado de urbanización } & Zona muy poblada & 44,71 & 47,45 & 36,56 \\
\hline & Zona media & 25,91 & 23,04 & 34,42 \\
\hline & Zona poco poblada & 29,38 & 29,51 & 29,02 \\
\hline \multirow{9}{*}{ Año } & Año 2005 & 11,94 & 12,23 & 11,06 \\
\hline & Año 2006 & 11,07 & 11,36 & 10,19 \\
\hline & Año 2007 & 10,76 & 11,17 & 9,56 \\
\hline & Año 2008 & 10,81 & 11,51 & 8,75 \\
\hline & Año 2009 & 11,25 & 11,78 & 9,67 \\
\hline & Año 2010 & 11,41 & 11,76 & 10,39 \\
\hline & Año 2011 & 10,81 & 10,61 & 11,41 \\
\hline & Año 2012 & 11,00 & 9,99 & 14,00 \\
\hline & Año 2013 & 10,94 & 9,58 & 14,98 \\
\hline \multicolumn{2}{|c|}{ Número de observaciones } & 18143 & 13570 & 4573 \\
\hline
\end{tabular}

Fuente: Encuesta de la Unión Europea sobre Condiciones de Vida (EU-SILC).

NURIA LEGAZPE MORALEJA es Profesora Contratada Doctora Interina en el Área de Economía Española e Internacional de la Universidad de Castilla - La Mancha (UCLM). Sus principales líneas de investigación abarcan estudios microeconómicos en los ámbitos de economía laboral y de la familia, en especial en la participación laboral de las mujeres y las decisiones de fecundidad. Entre las revistas donde ha publicado sus trabajos destacan Estudios de Economía Aplicada; Journal of Family Issues; Work, Employment and Society; Journal of Family and Economic Issues; Review of Economics of the Household; Papeles de Población y Revista de Economía Aplicada. 